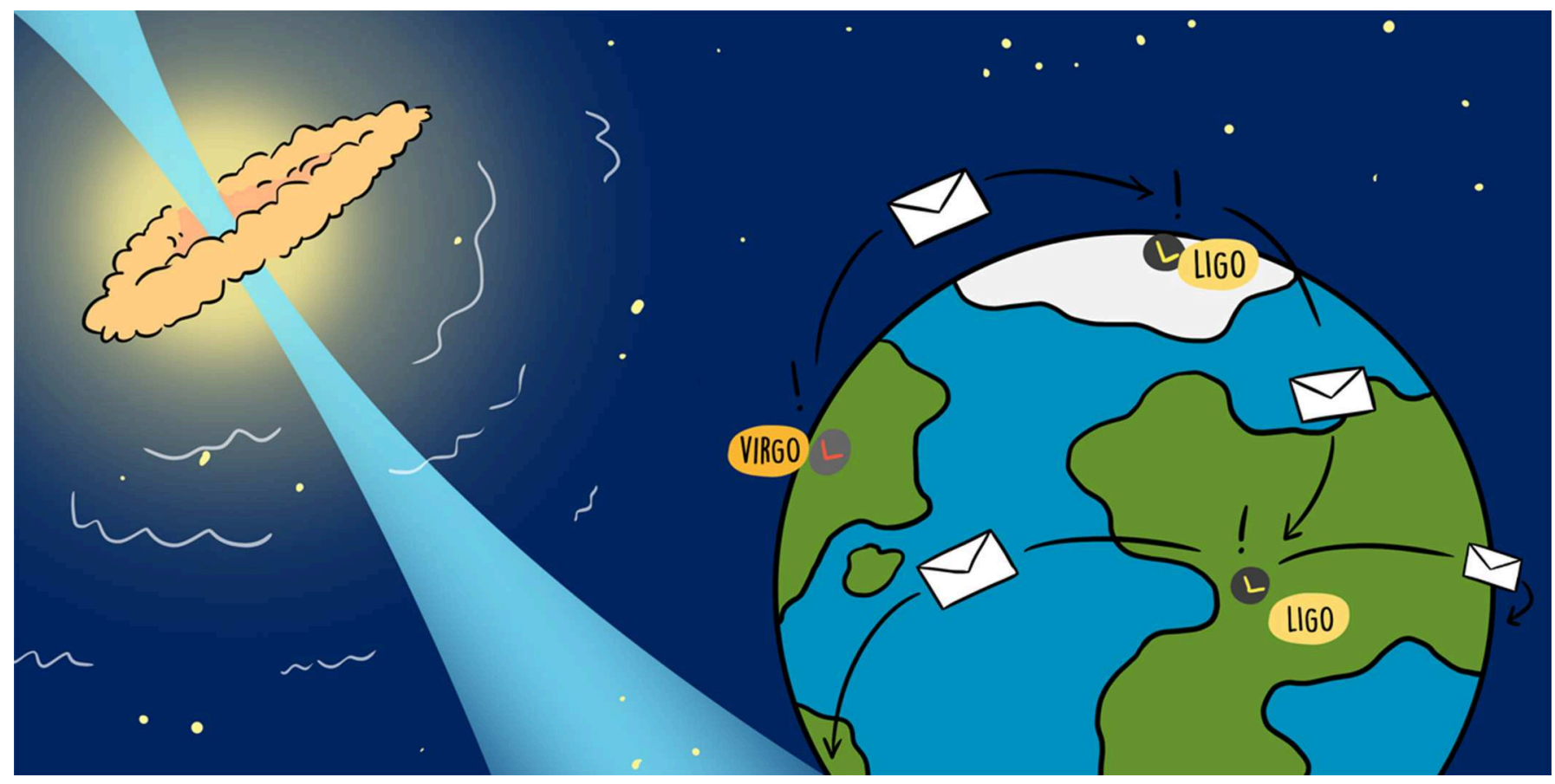

\title{
THE NEW ASTRONOMY: OBSERVING OUR UNIVERSE WITH LIGHT AND GRAVITY
}

\section{Joey Shapiro Key * for the LIGO Scientific Collaboration}

Physical Sciences Division, University of Washington Bothell, Bothell, WA, United States

YOUNG REVIEWER:

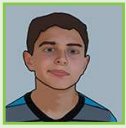

JONAH

AGE: 12
On a summer day in 2017, astronomers around the world received a message about an exciting collision of two stars far, far away. The message was sent by a team of astronomers from the LIGO and Virgo observatories. These new observatories are very different from the telescopes we have used to study our Universe up until now. LIGO and Virgo are gravitational wave observatories, listening for quiet ripples in spacetime created by the collisions of distant black holes and neutron stars. On August 17, 2017 LIGO and Virgo detected a signal that astronomers named GW170817, from the collision of two neutron stars. Less than two seconds later, NASA's Fermi satellite caught a signal, known as a gamma-ray burst, and within minutes, telescopes around the world began searching the sky. Telescopes in South America found the location of the collision in a distant galaxy known as NGC 4993. For the weeks and months that followed, astronomers watched the galaxy and the fading light from the collision. This is a new kind of multi-messenger astronomy where, for the first time, the same event was observed by both gravitational waves and light. 
Figure 1

Artist's illustration of the collision of two neutron stars, shown after they have collided in the center of the picture. The narrow beams represent the gamma-ray burst, while the rippling spacetime grid indicates the gravitational waves from the collision. Swirling clouds of material ejected from the merging stars are a possible source of the light that was seen by astronomers. Credit: National Science Foundation/LIGO/ Sonoma State University/A. Simonnet

\section{NEUTRON STAR}

The extremely dense object that remains after the collapse of a massive star.

\section{BLACK HOLE}

A region of spacetime, caused by an extremely compact mass, where the gravity is so intense it prevents anything, including light, from escaping.

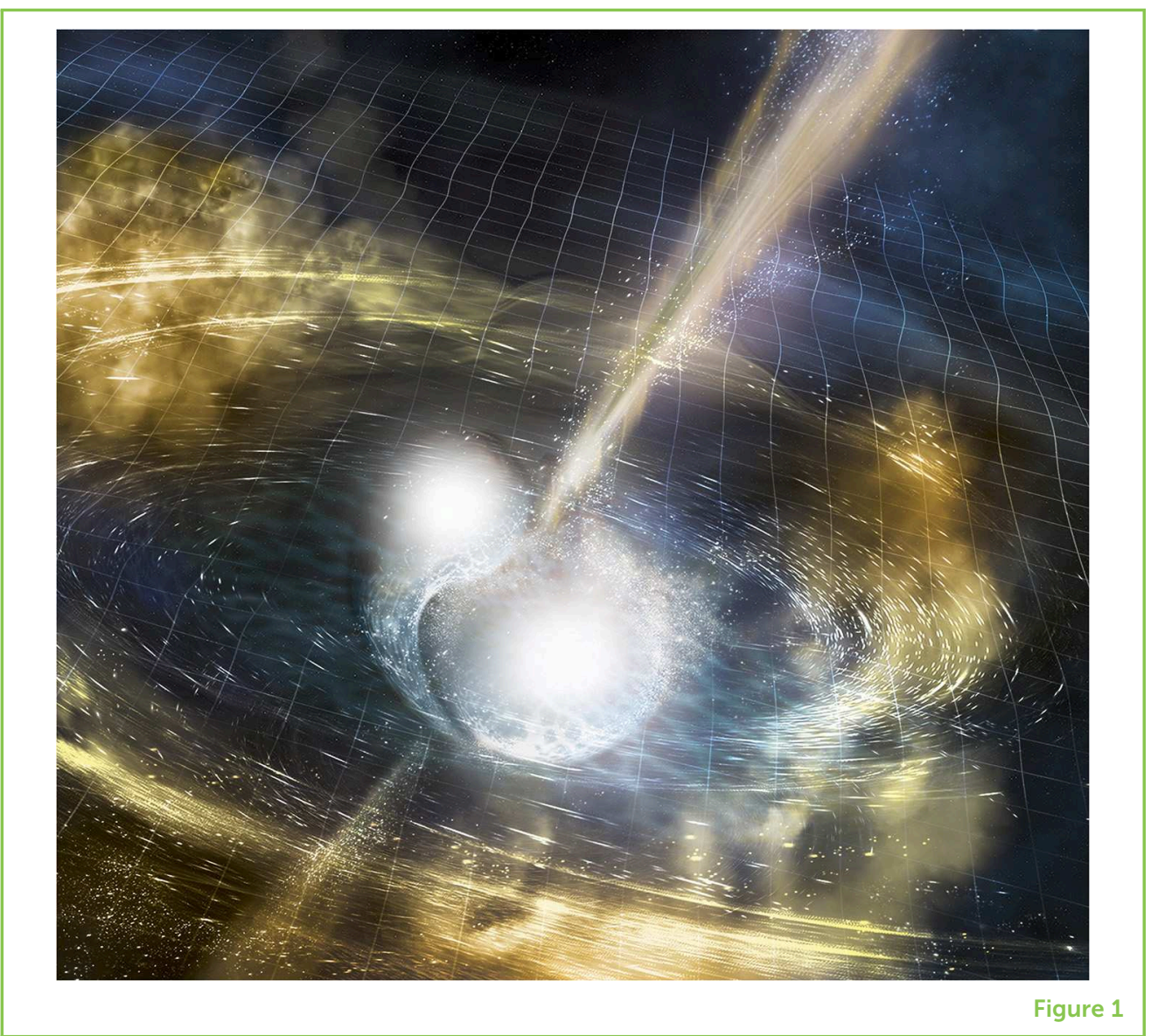

\section{NEUTRON STARS}

The stars in the night sky may seem like they have been there forever, but each star was created from gas and dust in space pulled together by gravity. A newly born star burns brightly until it runs out of fuel. Small- and medium-size stars like our own Sun end their lives as white dwarf stars, the glowing remains of the star's core. Stars much bigger than our Sun die a spectacular death, exploding as supernovae. The remains of a supernova explosion is a dense, dark core, either a neutron star or a black hole. The idea of a neutron star was first presented over 80 years ago, in 1934, but it was another 33 years before astronomers found a neutron star. In 1967, X-rays were detected from a distant neutron star and later the same year, the first radio pulsar was discovered. A pulsar is a highly magnetized neutron star that is spinning, sending a beam of radio pulse toward the Earth with each spin. Radio telescopes here on Earth can watch these pulses, which arrive like a steady ticking clock. Astronomers have also found binary neutron star systems, with two neutron stars orbiting around each other. When scientists planned to build the new LIGO and Virgo gravitational wave detectors, they hoped to find gravitational wave signals from some of these binary neutron star systems (Figure 1). 


\section{GRAVITATIONAL WAVES}

Over 100 years ago, Albert Einstein presented the Theory of General Relativity-a description of gravity that predicts black holes and curved spacetime. The theory also predicts gravitational waves, which are ripples in space and time that travel at the speed of light, created by the acceleration of massive objects, such as black holes and neutron stars. In September of 2015, the National Science Foundation's newly upgraded Advanced LIGO detectors observed the first gravitational wave signal from the collision of two black holes in a distant galaxy [1]. The event was named GW150914, for the gravitational wave (GW) signal detected in 2015 on September 14. The Laser Interferometer Gravitational wave Observatory (LIGO) detectors are located in Hanford, Washington and Livingston, Louisiana in the United States. Together with the European Virgo detector in Italy, they form a network of gravitational wave observatories that detected 10 separate gravitational wave signals from pairs of colliding black holes in their first two observing runs from 2015 to 2017. In the northern hemisphere summer of 2017, the observatories detected a new type of signal, one that came from the collision of two neutron stars [2].

\section{GAMMA-RAY}

The highest energy light, also known as electromagnetic radiation.

\section{GAMMA-RAY BURSTS}

Gamma-rays are a kind of light even more energetic than X-rays. In the mid-1960s, gamma-ray bursts (GRBs) were discovered by the Vela satellites. Astronomers later found that these GRBs came from space, but what could create such high energy gamma-ray bursts? Determining the sources of GRBs has been one of the key challenges in high-energy astrophysics ever since. In 2005, a short-duration gamma-ray burst (sGRB) was discovered to come from a distant galaxy and observations provided evidence that sGRBs might be the result of the collision of two neutron stars or the merger of a neutron star with a black hole. These very distant events are difficult to find, so it took a new kind of astronomy and the development of sensitive gravitational wave detectors to discover the neutron star collision that created the GW170817 gravitational wave signal and the GRB detected by the NASA Fermi satellite on August 17, 2017.

\section{A MULTI-MESSENGER DISCOVERY}

On August 17, 2017, NASA's Fermi satellite sent an automatic alert about a gamma-ray burst signal, now known as GRB170817A [3]. It took about $6 \mathrm{~min}$ for LIGO computers to find that a possible gravitational wave signal was detected at almost the same time at the Hanford observatory. The gravitational wave signal appeared to be from the collision of two neutron stars observed $2 \mathrm{~s}$ before the gamma-ray burst signal. LIGO and Virgo scientists issued an alert to astronomers around the world and, shortly after, they shared a map of 
Figure 2

To find the galaxy where the neutron stars collided, scientists combined the information from LIGO and Virgo, from Fermi, and from telescopes. In the circle representing a portion of the sky, on the left, the signal direction from LIGO is shown in light green, the combined LIGO and Virgo signal direction is shown in dark green, the direction from Fermi and the INTEGRAL satellite is shown in light blue, and the direction from the Fermi Gamma-ray Burst Monitor (GBM) is shown in dark blue. Telescopes searched the overlapping area of the sky and found a new bright source in the galaxy NGC 4993, shown with the small horizontal and vertical tick marks on the telescope image on the top right. The top box shows the galaxy from the Swope telescope $11 \mathrm{~h}$ after the discovery and the bottom box shows the galaxy from the DLT40 telescope 20 days before [3].

\section{NEUTRINO}

Tiny particle with no electric charge.

MULTI-MESSENGER ASTRONOMY

Using electromagnetic, gravitational-wave, and astro-particle data together to learn about the Universe.

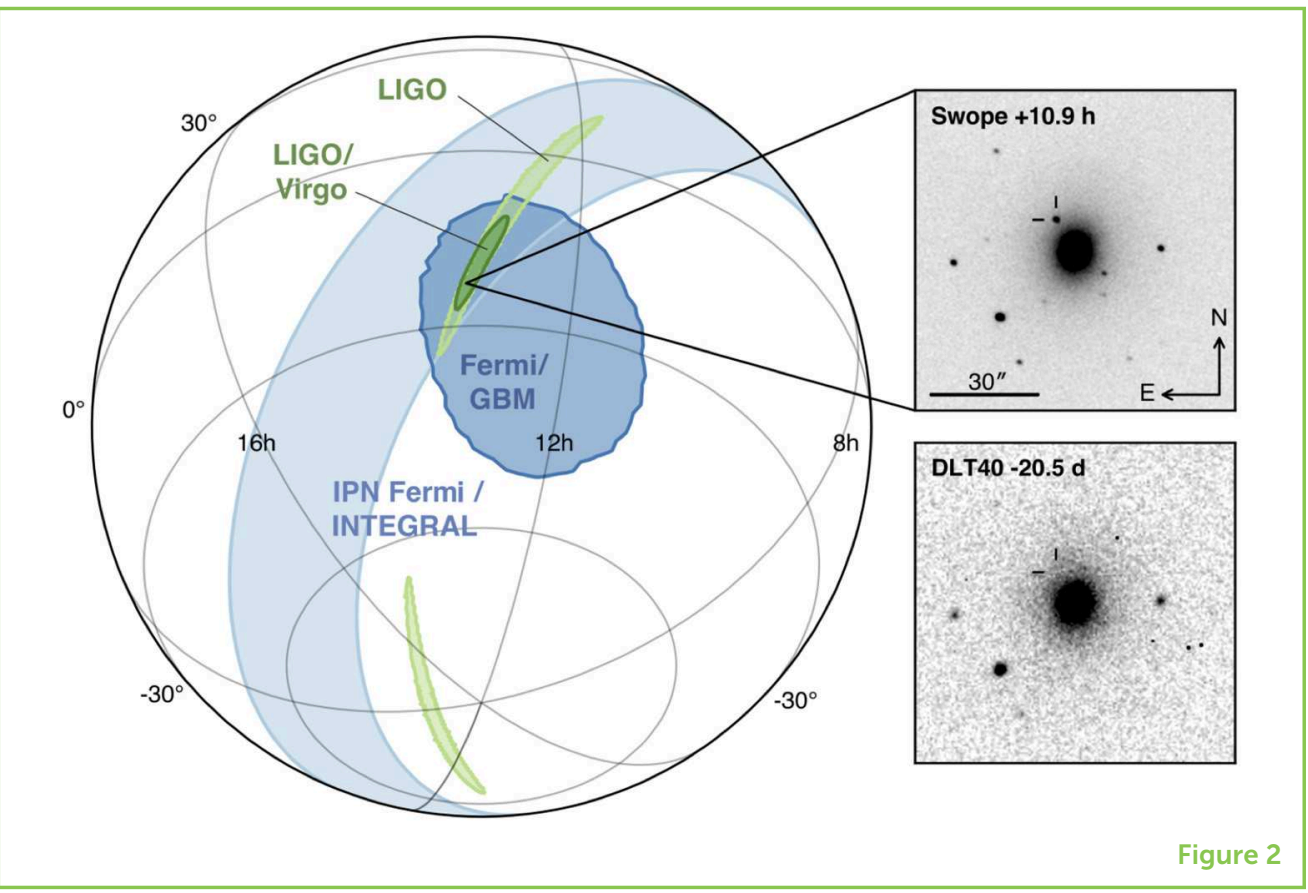

the area of the sky that was most likely the source of the gamma-ray burst and gravitational wave signals, shown in Figures 2, 3.

This event marked the first gravitational wave multi-messenger discovery: it was observed by both gravitational waves and light, which is also known as electromagnetic waves. At the time of the alert it was afternoon in the Western Hemisphere and, by nightfall, telescopes in South America were well-placed to search the sky for light from the collision. In the first few hours of darkness, a handful of telescope found a new bright source in the galaxy NGC 4993. Telescopes around the world turned to NGC 4993 to see what would happen next. Over the next 2 weeks, a network of ground-based telescopes and space-based observatories followed up on the initial detections. Observations were made in all the different kinds of light, with telescopes that could measure signals including ultraviolet, optical, and infrared light. Astronomers discovered that the new source of light was a kilonova, a bright short-lived event caused by the collision of two neutron stars. Following the kilonova, that part of the sky was watched with $\mathrm{X}$-ray and radio telescopes to better understand the collision. These observations revealed important information about the energy output of the explosion, the ejected material, and the environment of the collision. These observations showed us that neutron star collisions are able to create heavy elements, including gold, confirming what had only been a hypothesis before the measurement. Neutrino observatories searched without success for, high-energy neutrinos coming from the area of GW170817. It is a goal of multi-messenger astronomy to detect gravitational waves, electromagnetic radiation, and neutrinos from the same cosmic event [4]. Since the gravitational wave and gamma-ray burst signals occurred 
Figure 3

A sky map showing the direction of the GW170817 signal from combined LIGO and Virgo observations. Credit:

LIGO-Virgo/Cardiff U./C. North.

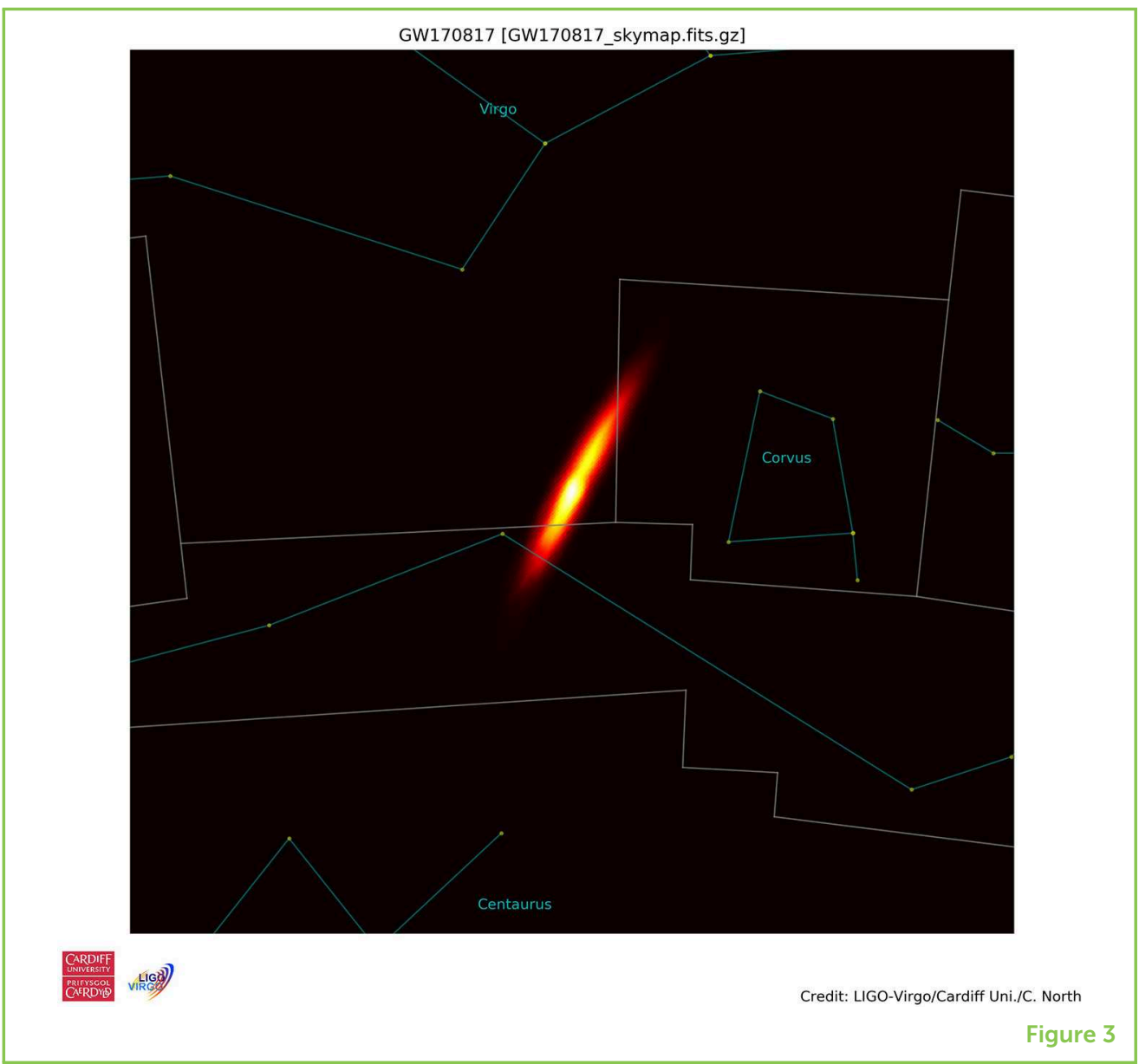

at nearly identical times, we now have confirmation of Einstein's prediction that gravitational waves and light waves travel at the same speed, over millions of kilometers.

\section{THE NEW ASTRONOMY}

The discovery of the gravitational wave signal GW170817 and the gamma-ray burst detected by the Fermi satellite on August 17, 2017 marked the first time both gravitational waves and light from a single astrophysical source had been observed. The LIGO and Virgo gravitational wave observatories sent an alert to astronomers around the world to search for light from the collision of two neutron stars. Telescopes found the location of the collision in a distant galaxy and, for the following weeks and months, astronomers watched and recorded the fading light from the collision. This event is the first time the same event was observed by both gravitational waves and light, showing how important it is for astronomers to work together to make new and exciting discoveries in a new era of multi-messenger astronomy. 


\section{ORIGINAL SOURCE ARTICLE}

Abbott, B. P., Abbott, R., Abbott, T. D., Acernese, F., Ackley, K., Adams, C., et al., LIGO Scientific Collaboration, Virgo Collaboration, Multi-Messenger Partners. 2017. Multi-messenger observations of a binary neutron star merger. Astrophys. J. Lett. 848:L12. doi: 10.3847/ 2041-8213/aa91c9

\section{REFERENCES}

1. LIGO Scientific Collaboration and Virgo Collaboration. 2016. Observation of gravitational waves from a binary black hole merger. Phys. Rev. Lett. 116:061102. doi: 10.1103/PhysRevLett.116.061102

2. LIGO Scientific Collaboration and Virgo Collaboration. 2017. GW170817: observation of gravitational waves from a binary neutron star inspiral. Phys. Rev. Lett. 119:161101. doi: 10.1103/PhysRevLett.119.161101

3. Abbott, B. P., Abbott, R., Abbott, T. D., Acernese, F., Ackley, K., Adams, C., et al., LIGO Scientific Collaboration, Virgo Collaboration, Multi-Messenger Partners. 2017. Multi-messenger observations of a binary neutron star merger. Astrophys. J. Lett. 848:L12. doi: 10.3847/2041-8213/aa91c9

4. LIGO Scientific Collaboration, Virgo Collaboration, ANTARES, IceCube, and Pierre Auger Observatory. 2017. Search for high-energy neutrinos from binary neutron star merger GW170817 with ANTARES, IceCube, and the Pierre Auger Observatory. Astrophys. J. Lett. 850:L35. doi: 10.3847/2041-8213/aa9aed

SUBMITTED: 25 January 2019; ACCEPTED: 06 September 2019;

PUBLISHED ONLINE: 01 November 2019.

EDITED BY: Shane L. Larson, Northwestern University, United States

CITATION: Key JS (2019) The New Astronomy: Observing Our Universe With Light and Gravity. Front. Young Minds 7:123. doi: 10.3389/frym.2019.00123

CONFLICT OF INTEREST: The author declares that the research was conducted in the absence of any commercial or financial relationships that could be construed as a potential conflict of interest.

COPYRIGHT @ 2019 Key. This is an open-access article distributed under the terms of the Creative Commons Attribution License (CC BY). The use, distribution or reproduction in other forums is permitted, provided the original author(s) and the copyright owner(s) are credited and that the original publication in this journal is cited, in accordance with accepted academic practice. No use, distribution or reproduction is permitted which does not comply with these terms. 


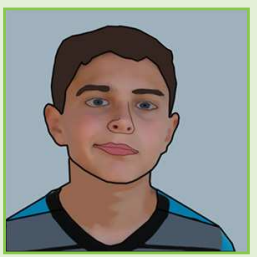

\section{YOUNG REVIEWER}

\section{JONAH, AGE: 12}

I love many different branches of science, which include physics, chemistry, programming, and mathematics. I like reading books and I am mostly a fan of fiction. I currently live in Northern California and I am in sixth grade. I spend time imagining many things and experimenting in a game called Minecraft.

\section{AUTHOR}

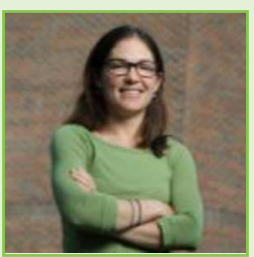

\section{JOEY SHAPIRO KEY}

Joey Shapiro Key is an Assistant Professor of Physics at the University of Washington Bothell. She has a B.A. in Astrophysics from Williams College and a Ph.D. in Physics from Montana State University. She is a member of the LIGO Scientific Collaboration. *joeykey@uw.edu 